\title{
A Blockchain-Based Deposit System to Reduce WEE
}

\author{
Sebastian Lawrenz \\ Institute for Software and Systems Engineering, \\ Clausthal University of Technology \\ Arnold-Sommerfeld-Straße 1 \\ 38678 Clausthal-Zellerfeld, Germany \\ SebastianLawrenz@tu-clausthal.de \\ Lukas Jacobs \\ Institute for Software and Systems Engineering, \\ Clausthal University of Technology, Germany \\ Arnold-Sommerfeld-Straße 1 \\ 38678 Clausthal-Zellerfeld, Germany \\ lukas.david.jacobs@tu-clausthal.de
}

\author{
Vera Stein \\ Institute for Software and Systems Engineering, \\ Clausthal University of Technology, Germany \\ Arnold-Sommerfeld-Straße 1 \\ 38678 Clausthal-Zellerfeld, Germany \\ vera.stein@tu-clausthal.de
}

\author{
Andreas Rausch \\ Institute for Software and Systems Engineering, Clausthal \\ University of Technology, Germany \\ Arnold-Sommerfeld-Straße 1 \\ 38678 Clausthal-Zellerfeld, Germany \\ andreas.rausch@tu-clausthal.de
}

\begin{abstract}
Nowadays, electronics/electrical appliances are becoming less and less appreciated. Due to ever shorter innovation cycles, especially in the field of consumer electronics, more and more electrical waste is being produced. Motivated by this fact we decided to face this challenge in a course called programming project course. There we introduced the problem and further the blockchain technology. The task was to design a blockchain-based solution to reduce Waste Electrical \& Electronic Equipment (WEE) and so to improve sustainability. The final idea here was to establish a deposit system for WEE. In this paper, we present the programming project from the development process of the first ideas to the completion of the running prototype and discuss the results.
\end{abstract}

\section{CCS Concepts}

-Computer systems organization $\rightarrow$ Architectures $\rightarrow$ distributed Architectures -Security and privacy $\rightarrow$ Systems Security $\rightarrow$ Distributed systems security -Software and its engineering $\rightarrow$ Software creation and management $\rightarrow$ Designing software $\rightarrow$ Software implementation planning $\rightarrow$ Software design techniques.

\section{Keywords}

Blockchain, Sustainability, Software Engineering

\section{INTRODUCTION}

Sustainability is one of the most praised topics in the social discourse of recent years. One of these reasons is the shifting of Earth Overshoot Day further and further forward. This day describes the date where humanity will have used nature's resource budget for the entire year. This day was already reached

Permission to make digital or hard copies of part or all of this work for personal or classroom use is granted without fee provided that copies are not made or distributed for profit or commercial advantage and that copies bear this notice and the full citation on the first page. Copyrights for third-party components of this work must be honored. For all other uses, contact the Owner/Author.

ICBCT'20, March 12-14, 2020, Hilo, HI, USA

(C) 2020 Copyright is held by the owner/author(s).

ACM ISBN 978-1-4503-7767-6/20/03.

https://doi.org/10.1145/3390566.3391686 last year on 29 July 2019 and in 2018 on 01 August [1].

Teaching software technology is not an easy task for universities. On the one hand, theoretical principles such as modeling and process models are of great importance, but on the other hand, they do not provide any practical experience. As a consequence of this, the Bachelor Students in computer Science of TU Clausthal have to complete a programming project course ( $p p c)$ in the fourth semester. In this course, students have to go through a complete software project in small groups. For the project, there is not a concrete task given, but a general challenge and a domain is given.

One of these general challenges in here was in the domain of sustainability and introduced the problem of Waste Electrical \& Electronic Equipment (WEE). WEE such as computers, TV-sets, fridges and cell phones is one the fastest growing waste streams in the EU, with some 9 million tons generated in 2005, and expected to grow to more than 12 million tons by 2020 [2]. Moreover, the disposal of WEE is often not correct (e.g. disposal of small electronic devices and batteries in residual waste) or partly even illegal (for example in woods or at public places).

Linked to this, we also gave a short introduction to the blockchain technology. Blockchain could be seen as a public ledger and all committed transactions are stored in a list of blocks and grows as new blocks are added to it continuously [3].

The students' task in the programming project course was finally to find a solution to reduce incorrect and illegal WEE, considering the Blockchain technology. This also leads to the following research question of this paper:

How can WEE reduced under consideration of the blockchain technology?

The rest of this paper is a combination of different perspectives. On the one hand from the perspective of the Product owner (the supervisors of the project programming course in this case) and on the other hand from the perspective of the development team (the students who took this project). In section two we will introduce the background, such as the problems of WEE, the ppc, and the blockchain technology. In Section 3 the first approach to tackle this problem will be introduced and discussed and Section 4 describes the final overall approach. In Section 5 the 
implementation will be shown, followed by a discussion in Section 6 . The paper ends with a final conclusion in chapter 7.

\section{BACKGROUND}

In this section, we are, going to introduce an important background for the topic. The first subsection deals with the problem of WEE and the section subsection describes the pcc in general and introduces also the methodology for the development process. Finally, we give a short overview of the blockchain and show some related work.

\subsection{Sustainability and WEE}

Consumers today have a traditional linear economy attitude (take, use, dispose), which is quite unsustainable. Due to ever shorter innovation cycles, especially in the field of consumer electronics, more and more electrical waste is being produced. For the year 2021, a production volume of more than 52 million tons of electronic scrap (WEE) is currently forecast worldwide [4]. In contrast, many European Union countries such as Germany, Denmark and Finland have a recycling rate of less than 50\% [5].

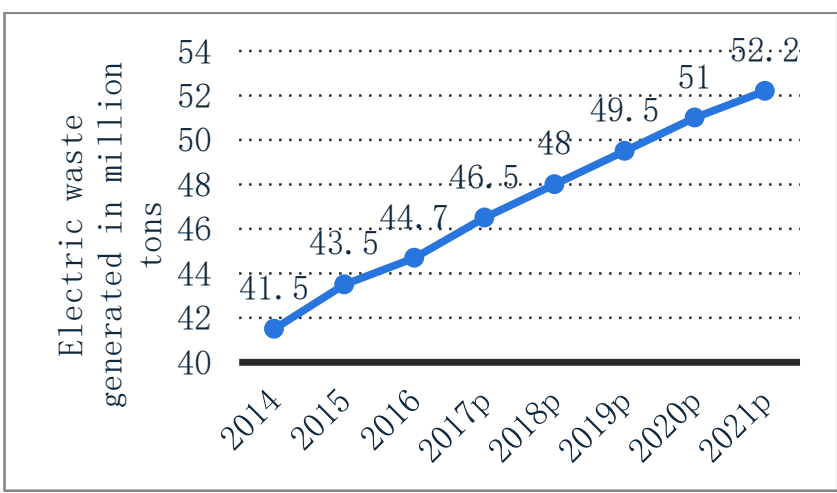

Figure 1. Electric waste generation worldwide from 2014 until 2016 and prognosis until 2021 [4]

One of the reasons for this low rate is the citizens' lack of awareness of the residual value of their equipment. In addition, some scrap is disposed of illegally or shipped to other countries. All this hinders more sustainable waste treatment systems and therefore the way to the circular economy. The circular economy is a regenerative system in which resource use and waste production, emissions, and energy waste are minimized by slowing down, reducing and closing energy and material cycles. To adopt a circular economy is one of the main goals of the European Union to achieve the sustainable consumption and production patterns that are one main aspect of the sustainable development goals as a part of the United Nations Agenda 2030 [6], [7].

Especially household WEE hold is a significant potential for better reuse and material recovery [4]. Here, in particular, is a special potential, as these devices are often not disposed of properly and therefore do not come back into the recycling cycle.

\subsection{Programming Project Course}

The programming project course is a bachelor course for students in the 4.th Semester of Computer Science at the Clausthal University of Technology. Within the programming project course, software development projects are assigned to groups of students. These groups realize, document and check their work in their respective projects in teamwork and present the results on a weekly basis according to scrum. This course has the following three learning objectives for the Students [8]:
1. Knowing the different roles of people involved in a software project

2. Understanding the specific tasks of these roles and being able to weigh them up against each other

3. Learning and training a sense of responsibility for the different tasks of the roles and working as a part of a team

One of these projects was already introduced in the first section and had the goal to develop a sustainable solution for the reduction of WEE under consideration of the blockchain technology.

The procedure of the course is as follows. In the first phase after the introduction of the topics, the students think about a possible solution and have to present them in front of the supervisors (called the Design phase). By the end of this first phase, they get feedback from all the supervisors and product owners which are part of the course. The rest of the course is organized agile according to Scrum [9]. The Scrum Master here is one supervisor for all the Student groups and the product owner is also a research assistant (one for every group). The students organize themselves independently as a development team and meet weekly with their product owner. They use a Kanban Tool (e.g. Trello) for the Sprint planning and as the product backlog. Every sprint has a duration and the sprint planning takes place together with product owner every week.

At the end of the semester, students have to deliver an executable prototype including the related documentation.

\subsection{Blockchain Technology}

Blockchain is a technology that has seen massive interest in the past few years. The general idea is, that everything happening depends on everything that has happened before. In general, a blockchain is a linked list of data. But, contrary to "classical" databases, it can make guarantees about the integrity of the stored data. The smallest unit of storage in a blockchain is called a transaction. This can be just about anything: information about a financial transaction, medical records, etc. One or more transactions are combined into a block, which consists of its transactions and some additional data, namely, it's cryptographic hash and the hash of the previous block. This creates a linked list of blocks, where each block points to its precursor using the precursors hash. Because the precursors hash is itself part of each block (and thus it's hash), any modification of a block will invalidate all following blocks, making it difficult to modify a blockchain unnoticed.

Most blockchains use proof of work to limit the number of blocks generated, mitigating the impact of denial of service attacks and data spamming. This is usually done by some kind of cryptographic puzzle, often by requiring a block's hash to have a set number of leading zeros, as laid out in the original bitcoin whitepaper by Satoshi Nakamoto [10].

The fact that most blockchain systems currently in use heavily rely on being essentially a form of distributed database is both an advantage and a disadvantage. Distribution makes the blockchain very robust against failures of an individual or even groups of nodes and the transparency of most blockchains makes it easy to discover any kind of irregular patterns. However, this also leads to quite a few privacy concerns in various widespread blockchain architectures. [11]. 
An idea often mentioned when talking about blockchain technology is smart contracts. These are programs that are part of a transaction on the blockchain. Probably the best-known use case of this is seen on the Ethereum blockchain, which is a cryptocurrency and has developed a special programming language for smart contracts, Solidity. These smart contracts get executed automatically, once certain criteria are met and are often used to transfer ether tokens between users. Smart contracts provide a very interesting way to have programs published immutably and executed within a given blockchain network [12],[13].

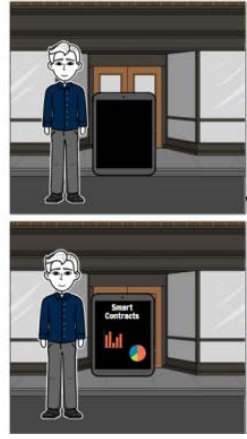

Upper row: Status Quo without the Recyclingchain
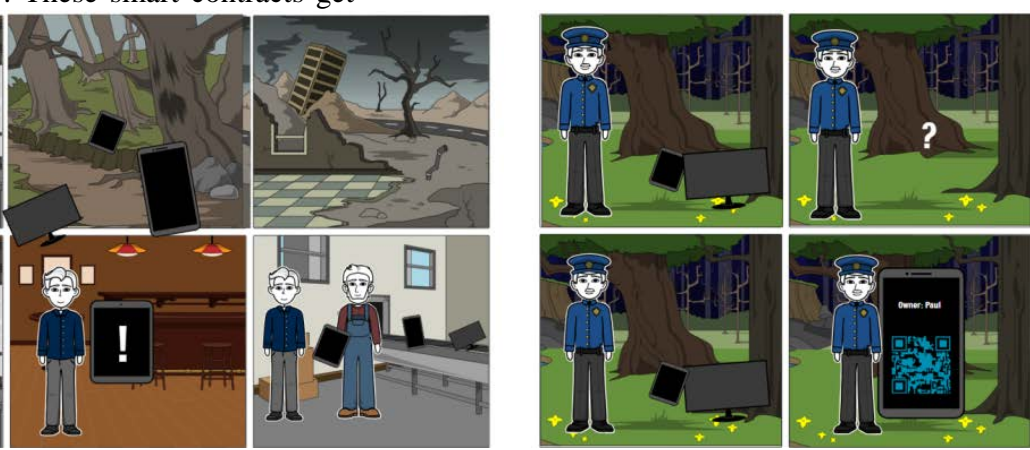

Figure 2. Sketch of the first RecyclingChain

\section{DESIGN PHASE}

As already introduced in the last section, the first phase of the pcc is just a design phase. After a short introduction about the problem with WEE and the blockchain technology, we made the first proposal for the final product. Thereby we identified the following two main problems, which we want to tackle within our approach:

1.) Illegal waste disposal (for example in the forest)

2.) The State cannot collect a fine because the owners cannot be identified

Based on this the first proposal was to assign an identification number to each device (comparable with the car chassis number of a car). The problem that this number could be made unrecognizable is avoided by using Smart Contracts. This is concluded when a device is purchased and is concluded between the State and the buyer. If the device is not properly disposed of after a certain period of time, a certain fee will be automatically collected. The contract will run in a blockchain and just needs the following information:

- $\quad$ Contract date

- Expiration date

- ID of the Device

- ID of the Owner

- ID of an authorized and verified Company

The ownership can change as well as that the expiration date can be extended. Verified companies are on the one side shops which sell EE Devices and on the other side recycling companies which confirm the professional return. A Sketch of this process (called RecyclingChain) is shown in figure 2. In the upper row of pictures, the actual situation without the Blockchain Solution is shown. Someone buys a device, uses it for a time and throws it away (shown in an overstated version) and the State official cannot figure out the owner. In contrast, the lower row shows the way with the recyclingChain. With the purchase of a new device, a contract is concluded which reminds you at some point of the disposal and finally, the owner brings it to a verified recycling company. If a device is thrown away illegal, the state official can figure out the owner and the owner pay a fine.

The feedback from the audience (the product owners and professors) criticized the problems according to the privacy policy

\section{Lower row: Process with RecyclingChain}

in Europe [14]. It is critical to store such personal data for a long period of time in a publicly viewable blockchain. Furthermore, the audience indicated that this solution is quite negative and would most likely be viewed negatively by the majority of society. We were asked to further develop the basic idea of this good solution, but to change its motivation towards a more positive way.

\section{OVERALL APPROACH}

In a second iteration based on the feedback from the design phase, we developed a new approach, which will be introduced in this Section.

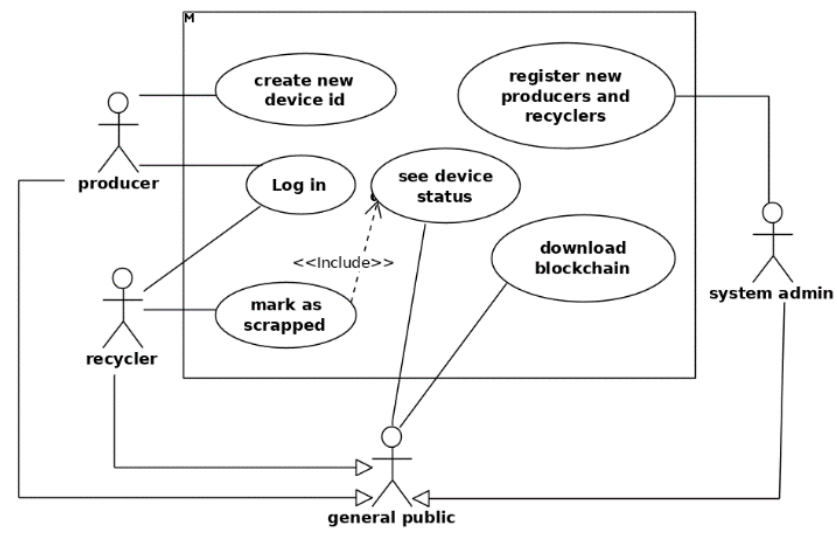

Figure 4. Use case diagram of the depositchain

We went from the RecyclingChain towards a DepositChain. The general idea of this approach is to create a deposit system for electrical devices, similar to the one that has already been achieved for plastic bottles in Germany [15]. Here customers have to pay a small fee when they buy a bottle and they will get it back by bringing the bottles back. Customers who buy a new electrical device, like a smartphone or a refrigerator, would have to pay a deposit, thus also strengthening the environmental awareness of the public. When the device stops working, it can be brought to a recycling yard and the deposit will be returned. Thereby, people who dispose of their electric scrap in a forest will have indirectly paid for cleaning the nature from it. 
As shown in the use-case-diagram (see figure 3), there are four kinds of stakeholders in the system: the general public, producer, recycling yard employees (recycler) and a system admin.

Each device will get a unique id and everybody who knows that id can enter it on a website or scan the QR-code to check if it is already marked as scrapped and how much deposit has been paid for it. This check should be executed every time the device is sold. The random unique ids will prevent the system from misuse like fake codes.

Only a producer can add a new device id and only the recyclers can mark a device as recycled. To use those special rights, they have to $\log$ in with a user-id, which is created by the system admin. Every process of creating a new id or marking a device as scrapped is saved in a blockchain that can be downloaded and backtracked by everyone, as there is no private data saved within. Therefore, it is possible to create a transparent deposit system for electrical devices.

\section{IMPLEMENTATION}

The main data is stored in a blockchain. A new block is added to the blockchain every time a new device id is added to the system or when a device is marked as scrapped. As shown in the domain model (see figure 4), each of these blocks contains a device id, the type of the device, the amount of many deposited for it (which of course varies by device type), the status of the device, whether it is already scrapped, a hash of the previous block and the creator id. The type of the device for example "smartphone" or "refrigerator" is for the people who recycle to control if the device id matches the device and therefore whether the amount of deposit is correct. The deposited amount of money and the status are saved in the block, where everybody is able to look it up, for example, if they want to sell it privately. No block can be manipulated, as this would invalidate all subsequent blocks, which are dependent on each previous block. Only creators can create a new block as they are the only ones, who have the private key to create a hash of the previous block. Each producer or recycler has a creator id that is saved in an object together with his username, password, company and public key.

The deposit system is based on the client-server-model, as shown in the structural view (see figure 5 left). The server-side (see figure 5 right) is programmed in python using the Django-

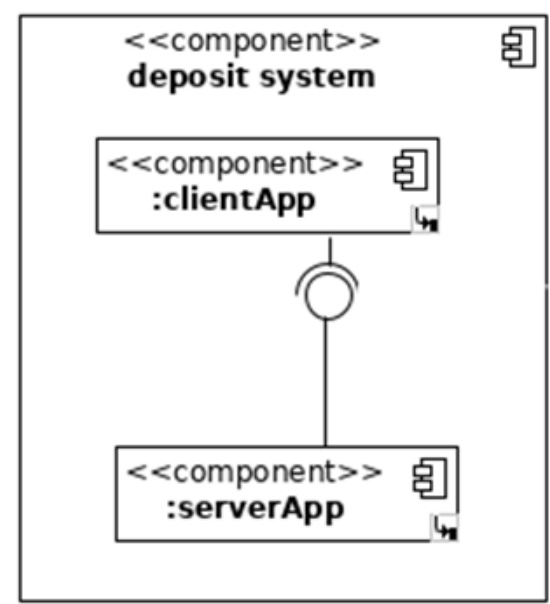

framework which is model-view-template based with a block and creator model. All of these instances are saved in an SQLitedatabase. The view is used to receive and answer HTTP requests of clients, to calculate hashes and to create new blocks. The templates are used to create a website. For the client site, an Android app was created with java which sends HTTP requests in JSON format to the server.

Therefore, a general person has two possibilities to check the amount of deposit of a special device. Firstly, entering the device id on the website and secondly, scanning the device QR-code with an app. They can also download the whole blockchain from the website. As it would be very costly for producers to create a unique code for every device manufactured manually, producers can also (and are encouraged to) do so via a REST-API, enabling the integration of the code generation process directly into their production lines. Recycling yard employees can log in in the app with their username and password and mark devices as scrapped that way.

The server implementation and the documentation can be downloaded at https://gitlab.com/tuc-isse/public/recyclingchain.

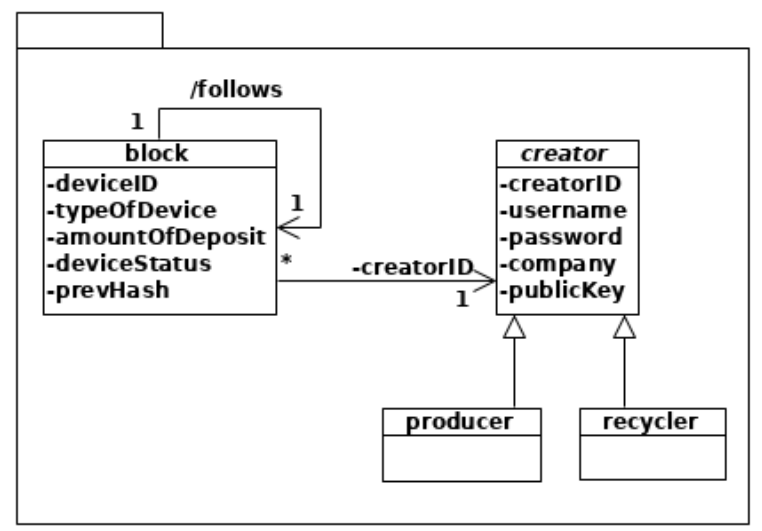

Figure 6.Domain diagram of the system

\section{DISCUSSION}

Blockchain has mostly been seen by the public in the context of cryptocurrencies. This has seen some limited real-world use, but in general blockchain as an industry and a technology is still

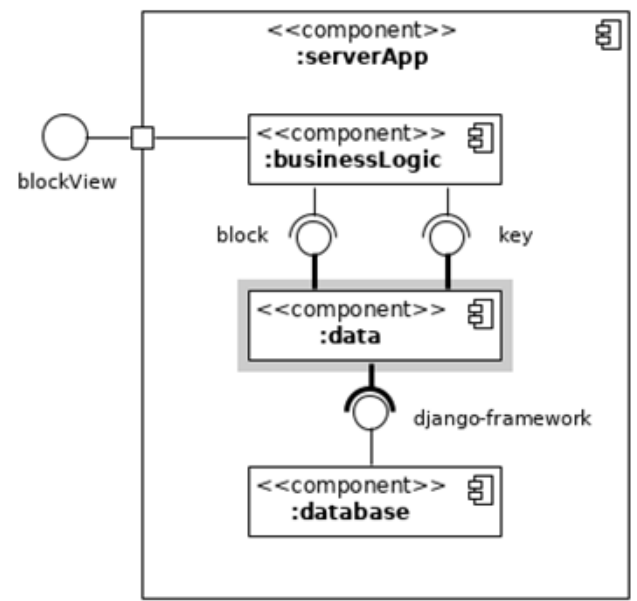

Figure 7. Component view of the system Left: Overall System Right: Server Application 
largely undefined. One of the biggest advantages of a blockchain is its immutability: each block is dependent on all previous blocks, thus tampering with anything that's already been written into a past block becomes almost impossible. We demonstrated that this can be used to set up a deposit system to incentivize proper recycling of electronic waste while balancing trust in the system through a transparent and easily verifiable, blockchain-based data storage on the one hand and the desire for anonymity, privacy, and high data protection standards (which are on the rise with legislation like the CCPA and the GDPR being enacted) on the other hand.

Admittedly our more centralized approach to blockchain technology is a bit contradictory to the ideology associated with blockchain by many of its advocates, but it also provides a way of using blockchain technology to government entities, which often have regulations in place prohibiting a more decentralized network. Through the usage of asymmetric cryptography, we enable more centralized storage of the block data itself, while preventing manipulation of the blocks, as the storage agency does not possess the private keys used to generate the blocks.

Another advantage of a system based on RSA and a proof-ofstake architecture (rather than the usual proof-of-work) is its speed: Bitcoin, for example, has a block generation time of 10 minutes currently, with a block size of $1 \mathrm{MB}$ and an average transaction size of 334.58 bytes [16], thus Bitcoin is only capable of 5.22 transactions per second, globally (see Formula 1). Those 5.22 transactions per second come at the cost of an enormous use of electricity. Our proof-of-stake architecture enables far better scaling while being much more ecologically sustainable.

$$
\frac{\left(\frac{\text { BlockSize }}{\text { TransactionSize }}\right)}{\text { SecondsPerBlock }}=\frac{\left(\frac{1048576 \mathrm{~B}}{334.58 \mathrm{~B}}\right)}{600 \mathrm{~s}}=5.22 \mathrm{~Hz}
$$

\section{CONCLUSION}

In this paper, we showed the possibility to implement a deposit system based on the core ideas of blockchain-technology. The system was implemented prototypically during the ppc by a group of students and demonstrates an easy and adaptable solution. The main goal of this paper to develop a solution for the reduction of WEE was achieved. By using a proof-of-stake algorithm instead of proof-of-work we also presented a sustainable solution in terms of energy consumption.

\section{ACKNOWLEDGMENTS}

This research has been funded by the Federal Ministry of Education and Research of Germany in the framework of EffizientNutzen (project number 033R240E). Furthermore, we would like to thank Phillip Erber, Elisabeth Fittschen and Julia Lanfer for participating in the ppc and be part of the development team. Also, we would like to thank Dirk Herrling for the organization of the ppc and for being the Scrum Master.

\section{REFERENCES}

[1] E. O. Day, "About earth overshoot day," Hentet fra https//www. overshootday. org, 2019. [Online]. Available: https//www.overshootday.org [Accessed: 12-Jan-2020].

[2] "Waste electronic equipment - Environment - European Commission.” [Online]. Available: https://ec.europa.eu/ environment/waste/weee/index_en.htm. [Accessed: 12-Jan2020].
[3] Zheng, Z., Xie, S., Dai, H., Chen X. and Wang, H. “An Overview of Blockchain Technology: Architecture, Consensus, and Future Trends," Proc. - 2017 IEEE 6th Int. Congr. Big Data, BigData Congr. 2017, pp. 557-564, 2017.

[4] Statista, \& United Nations University. "Erzeugung von Elektroschrott weltweit in den Jahren von 2014 bis 2016 und eine Prognose bis 2021 (in Millionen Tonnen)“ [Graph]. In Statista, 2017 [Online available] https://de.statista.com/ statistik/daten/studie/792541/umfrage/erzeugung-vonelektroschrott-weltweit/ [Accessed: 13-Jan-2020].

[5] Eurostat. "Recyclingquote von Elektroschrott in der Europäischen Union (EU-28) nach Ländern im Jahr 2017“ [Graph]. In 2019 [Online available] https:/de.statista.com/statistik/daten/ studie/795452/umfrage/recyclingquote-von-elektroschrott-ineu-laendern/ [Accessed: 13-Jan-2020].

[6] Resolution adopted by the General Assembly on 25 September 2015: A/RES/70/1, 2015. [Online available] https://www.un.org/en/development/desa/population/migrati on/generalassembly/docs/globalcompact/A_RES_70_1_E.pd f [Accessed: 13-Jan-2020].

[7] European Commision, “An EU action plan for the circular economy,” Com, vol. 614, p. 21, 2015.

[8] Modulhandbuch für den Studiengang B.Sc. Informatik/Wirtschaftsinformatik p. 74, 2019 [Online available] https://www.tu-clausthal.de/fileadmin/ TU_Clausthal/dokumente/Studiengaenge/Modulhandbuecher /MHB-B-IW-3S-19-02.pdf [Accessed: 13-Jan-2020].

[9] Schwaber, K. and Sutherland J. “The Scrum Guide”, 2017 [Online available] https://www.scrumguides.org/ docs/scrumguide/v2017/2017-Scrum-GuideUS.pdf\#zoom=100 [Accessed: 13-Jan-2020]

[10] Nakamoto, S. "Bitcoin: A Peer-to-Peer Electronic Cash System.” Manubot, 2019 [Online available] https://git.dhimmel.com/bitcoin-whitepaper/ [Accessed: 13Jan-2020]

[11] Halpin, H. and Piekarska, M. "Introduction to security and privacy on the blockchain," Proc. - 2nd IEEE Eur. Symp. Secur. Priv. Work. EuroS PW 2017, pp. 1-3, 2017

[12] Szabo, N. "Smart contracts: building blocks for digital markets," EXTROPY: The Journal of Transhumanist Thought,(16) 18, 1996.

[13] Antonopoulos, A. M. and Wood, G. Mastering ethereum: building smart contracts and dapps. O’Reilly Media, 2018.

[14] Voigt, P. and Bussche, A. "The eu general data protection regulation (gdpr)." A Practical Guide, 1st Ed., Cham: Springer International Publishing, 2017.

[15] Souren R. and Hartlep, U. Recycling von Einweggetränkeverpackungen in Deutschland: Gesetzliche Regelungen und Funktionsweise des implementierten Pfandsystems, vol. 2, no. January. 2011.

[16] Historical Bitcoin Data, [Online]. Available: https://tradeblock.com/bitcoin/historical/1h-f-tsize_per_avg01101 [Accessed: 12-Jan-2020]. 